\section{Deutsches Institut für Normung e.V. (DIN)}

\author{
A. M. Gressner ${ }^{1}$ und O. A. Gressner ${ }^{2}$ \\ ${ }^{1}$ Labor Dr. Wisplinghoff Berlin, Berlin, Deutschland \\ ${ }^{2}$ Labor Dr. Wisplinghoff und Kollegen Köln, Köln, \\ Deutschland
}

Definition Das Deutsche Institut für Normung (DIN) bietet rund um die zentrale Dienstleistung der Normung eine Reihe weiterer Leistungen an, die den Zugang zur Normung und zu Normungsverfahren, zu Normen und Norminhalten erleichtern.

Beschreibung Das im Jahr 1917 als Normenausschuss der deutschen Industrie (NADI) gegründete Institut führt die fachliche Arbeit der Normung in Arbeitsausschüssen bzw. Komitees durch. Für eine bestimmte Normungsaufgabe ist jeweils ein Arbeitsausschuss bzw. ein Komitee zuständig, die zugleich diese Aufgaben auch in den regionalen und internationalen Normungsorganisationen wahrnehmen. Im Regelfall sind mehrere Arbeitsausschüsse zu einem Normenausschuss im DIN zusammengefasst, von denen es derzeit 76 gibt. Für die $>$ Klinische Chemie und $>$ Laboratoriumsmedizin sind folgende Normenausschüsse von besonderer Relevanz:

Normenausschuss „Laborgeräte und Laboreinrichtungen (FNLa) " FnLa erarbeitet Normen für Geräte und Einrichtungen in chemischen, physikalischen, biologischen und medizinischen Laboratorien. Die Normen behandeln die Genauigkeit von Messgeräten, das Zusammenpassen von Glasgeräten und Einrichtungen, die nationale und internationale Verständigung durch einheitliche Terminologie sowie Funktionen, Sicherheit und Prüfung von Laborausrüstungen aller Art.
Durch die Beteiligung der Arbeitsausschüsse an Forschungsvorhaben können neueste Erkenntnisse in die Normen einfließen. Die Geschäftsstelle des Normenausschusses führt gleichzeitig die Sekretariate für die CEN/TC 332 „Laboratory Equipment“ und ISO/TC 48 „Laboratory Glassware and Related Apparatus", was eine weitere Vereinheitlichung des nationalen, europäischen und internationalen Normenwerkes für Laboratorien ermöglicht.

Normenausschuss „,Medizin (NAMed) “ NAMed ist zuständig für die nationale Normung auf den Gebieten Medizinprodukte, Transfusion, Infusion, Injektion, Laboratoriumsmedizin und Klinische Chemie, Medizinische Informatik, Medizinische Mikrobiologie und Immunologie, Sterilisation, Desinfektion, Sterilgutversorgung u. a. Die Maßnahmen auf den genannten Gebieten werden mit dem Ziel durchgeführt, ein möglichst hohes Qualitätsniveau der genormten Produkte und Verfahren festzuschreiben, um damit einen wichtigen Beitrag für die Patientensicherheit zu leisten. Normen und Normeninhalte und weitere Ergebnisse der DIN-Arbeit bietet das DIN in der Regel über den Beuth-Verlag an (Beuth-Verlag GmbH, Burggrafenstraße 6, D-10787 Berlin, Tel.: 03026010 , Fax: 030 26011260, E-Mail: postmaster@beuth.de, Internet: www.beuth.de).

\section{Adresse:}

DIN Deutsches Institut für Normung e.V.

Burggrafenstr. 6

D-10787 Berlin

Tel.: 030-260-10

Fax: 03026011231

E-Mail: postmaster@din.de

Internet: www.service.din.de 